\title{
The Abstracts of the 4th UQ Symposium on Organisational Psychology
}

\section{July 2004}

\section{Emmanuel College, University of Queensland}

\section{Organised and sponsored by the Centre for Organisational Psychology, University of Queensland}

\author{
Abstracts Editor: Robin Martin \\ University of Queensland
}

\begin{abstract}
These abstracts form the collection of papers that were presented at the 4th UQ Symposium on Organisational Psychology held at Emmanuel College, University of Queensland, Brisbane on Saturday 24th July, 2004. The UQ Symposium on Organisational Psychology is an annual even organised by the Centre for Organisational Psychology at the University of Queensland. The aim of the symposium is for academic psychologists to present their latest research to fellow academics and practitioners. Papers were accepted for either paper presentation or poster presentation following a peer-review process. The 100 delegates who attended consisted of practitioners and academics. The invited speaker was Dr Sally Carless (Monash University, Melbourne) who presented her latest research on selection procedures. The 5th Symposium will take place on Saturday 4th June, 2005. For more information about the UQ Symposium on Organisational Psychology series please contact Robin Martin (r.martin@psy.uq.edu.au).
\end{abstract}

Robin Martin 
Gaining benefits from career climate: The role of career commitment

AREND, S., \& MARTIN, R. (University of Queensland). arends@psy.uq.edu.au

B usiness contexts have changed considerably over the last two decades forcing many organisations into major changes (restructuring, downsizing). This has affected people's work lives, especially their organisational careers. Individual's careers now occur across different organisations, replacing the 'one employer for life' tradition. Personal career commitment has become an important concept in explaining how people operate within this dynamic work context, suggesting that career committed employees evaluate their organisation in terms of the quality of its career management practices ('career climate'). The perceived quality of the organisation's career management practices affects the types and strength of employees' organisational commitment. Three samples with people from different professional backgrounds were used. In line with hypotheses, results showed that, across the three samples, personal career commitment and 'career climate' are strong predictors of all components of organisational commitment (affective, normative, and continuance commitment: i.e., low alternatives and high sacrifice). Additionally, career commitment moderated the relationship between career climate and affective commitment, high sacrifice and low alternatives. These results highlight the importance of individual career aspirations and commitment. Organisations need to provide effective career management practices as a means to raise employees' organisational commitment and to derive the benefits of a committed workforce.

\section{Espoused organisational values and SARS: When actions speak louder than words}

ARNOLD, S. (University of Queensland), \& BOYLE, M. (Griffith University).

S.Arnold@business.uq.edu.au

tatements of an organisation's values or code of Oethics have become increasingly popular in recent times. Yet, little research has been done into how employees perceive, and react to, the values that are espoused by their organisation. We contend that the values espoused by an organisation should be most salient to its employees in times of organisational crisis. In the current study, we investigated employee experiences during an organisational crisis, in an organisation that has a clearly defined and promoted set of organisational values. We interviewed thirty-three employees who worked in a
Singaporean hospital during the 2003 crisis due to the Sudden Acute Respiratory Syndrome (SARS) virus. Qualitative analysis of the data revealed that interviewees were unable to consistently describe the hospital's values, their meaning, or their influence during the crisis. However, the employees perceived that the hospital's actions during the crisis were consistent with the notion of a general valuesbase. Furthermore, individuals were inspired by the values-based organisational actions to carry out organisational citizenship behaviours of their own. The implication from this study is that the specific wording of a values statement is less important to employees than their perception that the organisation acts in a values-based manner.

\section{Development of a measure of job passion}

ASHKANASY, NM. (University of Queensland), CADY, $S$. (Bowling Green State University, USA),

\& NOORDINK, P. (University of Queensland).

P.Noordink@business.uq.edu.au

Tob ob passion is frequently referred to in the popular literature, but research into this construct has been restricted because of the unavailability of a reliable and valid measure. The emphasis of research in industrial and organisational literature appears to be directed primarily at increasing productivity and profits, rather than advancing insight into why workers love or dislike jobs. We address this issue through development of a new 18-item scale. The scale was first pilot tested on an undergraduate population, then further tested on a sample of 255 employees from various organisations, though mainly from the university sector, and from a human services department in local government. Three dimensions were identified: affective passion, job-before-leisure, and performance-focus. Reliability of the overall scale was good ( $\alpha=.86$ ), as it was with the three subscales: $.83, .69$, and .75 for affective passion, job-beforeleisure, and performance-focus respectively. The validity of the job passion measure was determined against measures of workaholism, job satisfaction, burnout, organisational commitment, and organisational citizenship behaviour.

\section{Crosscultural factors influencing performance management in organisations: A research agenda}

BAILEY, C. (Queensland University of Technology). c2.bailey@qut.edu.au

$\mathrm{W}$ th a rise in internationalisation and globalisation, organisations are facing the challenge of implementing processes which are both appropriate 
and effective across their various geographical locations (Groeschl, 2003). One such process is the management of employee performance. Regarded by the vast majority of organisations as a central activity for employees at all organisational levels (Redman, 2001), the Performance Management (PM) interventions utilised are critical to both individual wellbeing and organisational productivity (Fletcher, 2000). Since the seminal work of Hofstede (1984), research into crosscultural differences has grown steadily. Similarly, much has been written about performance appraisal, and its various constituent parts (e.g. purpose, methods, type of appraisers and impact on performance). However, to date only a handful of studies have actually linked the two, investigating the impacts of cultural diversity within the appraisal process. From a review of this literature, this poster presents a synopsis of key issues, linking findings across cross-cultural and PM research. A theoretical model is proposed, providing hypotheses as to the nature and impact of crosscultural effects for each aspect of the Performance Management process (e.g., appraisal objectives, methods, choice of assessors). The practical implications for organisations implementing a global PM process are also outlined.

\section{Turnover and training: The influence of perceived organisational support}

BANKS, T., YEO, G., \& NEAL, A.

(University of Queensland).

t.banks@qut.edu.au

$\mathrm{T}$

The purpose of this study was to ascertain whether perceived organisational support moderated the relationship between turnover intention and training participation. The sample comprised 4316 employees in 2002 and 5757 employees in 2003, from the State Government Public Service. Data were collected via surveys. Multiple hierarchical regressions were conducted. The findings offer limited support for the hypothesised moderating influence of perceived organisational support. The interaction between perceived organisational support and training participation did significantly contribute to the prediction of turnover in time 1 and time 2. Furthermore, as anticipated, training participation was negatively related to turnover intention and this relationship was stronger in climates were organisational support was perceived to be low rather than high. However, longitudinal analysis revealed that given the effect of other variables, the interaction between perceived organisational support and training participation, did not significantly contribute to the prediction of turnover intentions. An implication of these findings is that employers who are striving to manage turnover, should focus on managing perceived organisational support rather than training participation.

\section{How managers enhance employee outcomes: The role of recognition}

BLOCH, AC., \& MARTIN, R. (University of Queensland). bloch@psy.uq.edu.au

$M$ anagers can motivate their employees through their behaviours and interactions with them. Leader behaviour that recognises accomplishments from employees may be critical in influencing employee role perception and work related outcomes. Such leader behaviours include actions that facilitate accomplishments and support subordinates efforts and involvement (House, 1971). By providing feedback and recognising employees, managers may highlight the link employees perceive between achievements and rewards (Naylor, Pritschard, \& Ilgen, 1980). Even though one of the most basic principles of management is that organisations cannot function effectively unless managers recognise behaviours that support organisational goals (Kerr, 1975), empirical research in the academic literature on the nature, function and consequences of recognition is still scarce. Therefore the purpose of the current research was to extend prior research by investigating the effects of managerial recognition on goal commitment, efficacy beliefs and employee wellbeing. Managerial recognition was defined as 'personal attention, mostly conveyed verbally, through expressions of interest, approval, and appreciation for a job well done' (Luthans \& Stajkovic, 2000). It was found that employees who receive approval from their manager have positive perceptions of the groups in which they work, are very committed to their assigned goals and experience high levels of wellbeing.

\section{Managing occupational stress: The state of play}

BROUGH, P. (Griffith University).

$\mathrm{D}$ espite fifty years of active research into the causes and consequences of psychological stress, coping behaviours and wellbeing outcomes, there remains considerable debate in this field. Discussions continue on the optimum definitions, measurement, analysis and causal relationships of these concepts. This presentation will provide an overview of these current discussions and will attempt to identify the most fruitful directions for both future research and for the organisational management of occupational stress. The discussions will be exemplified by recent examples of applied research. 


\section{Development of the student stress scale}

BROUGH, P., CHASE, R., \& REYNOLDS, S. (Griffith University).

p.brough@griffith.edu.au

$\mathrm{R}$ ecent developments in student experiences, relating primarily to student loans, university fees and generally diminished student-staff ratios are expected to have an adverse impact upon the quality of student life. This paper identifies the sources of contemporary undergraduate student stress. The development of a student stress measure is described and was found to consist of five components: academic demands, financial demands, external demands, academic confidence and future expectations. The scale was deemed to have generally acceptable psychometric properties and was tested for associations with measures of individual differences, coping behaviours and psychological well-being, in two individual student samples. Financial demands was identified as one of the most common student stressors and as the strongest individual predictor of psychological well-being. The application of this research within the universities in terms of the provision of suitable student support systems and for the future growth of vocationally orientated academic psychology programs is discussed.

\section{Keeping people at work: Evaluating the type of supervisor support on work-related psychological outcomes}

BROUGH, P., \& PEARS, J. (Griffith University). p.brough@griffith.edu.au

$\mathrm{T}$ This investigation evaluated the influence of practical and emotional types of workplace support upon occupational stress, job satisfaction and psychological wellbeing. A sample of human services workers responded to a self-report questionnaire. Both emotional and practical supervisor support directly predicted job satisfaction but did not significantly predict psychological wellbeing. Colleague social support was not significantly predictive of either outcome. Post-hoc analyses revealed the mediating role of job control and the partially mediating role of both emotional and practical supervisor support on job satisfaction. The importance of supervisor support for the maintenance of positive work attitudes for the operational employees in particular, is discussed.
Workplace violence experienced by paramedics: Its influence on social support, job satisfaction and psychological strain

BROUGH, P., \& ULUICICIA, M. (Griffith University). p.brough@griffith.edu.au

$\mathrm{T}$ he increase in the incidents of workplace violence perpetrated against paramedics (ambulance officers) is of growing concern and has resulted in revisions of organisational support, training and operational practices. The current research investigates the types of violent incidents experienced by 120 Australian paramedics during their operational duties. Both verbal and physically violent incidents were commonly experienced. These occupational experiences directly predicted adverse levels of job satisfaction and indirectly predicted psychological strain. A perceived lack of supervisor social support increased the experiences of workplace violence. Organisational implications focusing on training provisions and recommendations for future research in this area are discussed.

\section{The impact of selection procedures and interviewer characteristics on applicants}

CARLESS, S. (Monash University).

$M$

y talk will explore the effects recruitment and selection procedures have on applicants. The first issue I will examine will be the impact of selection procedures, (psychological tests, physical agility tests and interviews) on applicant perceptions of fairness, attraction to the organisation and job acceptance intentions. This issue will be explored with two samples: police force applicants and graduate applicants for a telecommunications company. The next issue I will look at will be the joint effects of selection procedures and job and organisational characteristics on applicant attraction and acceptance intentions. It is argued that testing the impact of selection procedures together with job and organisational characteristics provides a stronger test of the effects of selection procedures. The final issue that I will examine will be the impact of recruiter characteristics on applicants. A model will be proposed that recruiters have a direct and indirect effect on applicant attraction and acceptance intentions. The sample for this study was graduate applicants for an international bank. 
Does gender influence the experience of stress? An examination of police and organisational stressors reported by Australian police officers

DREW, JM. (Queensland Police Service), \& THOMPSON, B. (Griffith University). b.thompson@griffith.edu.au

W omen police may experience more stress emanating from operational police duties (Brown \& Fielding, 1993) than male officers. The impact of gender on police-specific stress, that is stress resulting from operational police duties such as danger and the use and discharge of firearms in the line of duty, and organisational stressors such as the physical organisational environment, supervision, co-workers and administration, was examined in a large sample. Six hundred and fifty male, and 322 female police officers, employed in an Australian police organisation completed an adaptation of the Hart, Wearing \& Headley (1993) Police Daily Hassles Scale. A MANOVA with gender as the independent variable, and operational and organisational stress scales as dependent variables, indicated that gender did not significantly influence levels of stress emanating from operational police events, or the physical organisational environment and resources. However, compared to female officers, male officers reported significantly higher levels of stress resulting from such organisational factors as supervision, coworkers and administration. Thus female officers are no more likely than male officers to experience operational stress than their male colleagues, and experience less stress from colleagues and administration.

\section{The effects of teacher burnout and organisational citizenship behaviour on students' quality of school life: A multilevel perspective}

HANNAM, R., JIMMIESON, N., \& YEO, G. (University of Queensland). hannam@psy.uq.edu.au

$\mathrm{T}$ This study investigated the negative impact of teacher burnout and the positive impact of teachers' organisational citizenship behaviours (OCBs) on primary school students in Years 4-7. Teachers $(N=171)$ were surveyed using the Maslach (1980) Burnout Inventory and the effects of emotional exhaustion, depersonalisation, and personal accomplishment on student perceptions were examined. In addition, a new scale was developed to assess three types of teacher OCBs (helping students, civic virtue, and professionalism) that potentially impact students. Student perceptions were assessed using the Quality of School Life survey (QSL; Ainley \& Bourke, 1992) which contains six sub-scales. Based on previous findings about burnout and its impact on clientele, we predicted that students $(N=3058)$ would report a lower quality of school life when their teachers reported more burnout. We also hypothesised that students whose teachers engaged in more OCBs would report greater satisfaction with school. Overall, we found moderate support for both sets of predictions. Multilevel analyses indicated that a significant proportion of variance in student perceptions was attributable to classroom factors as opposed to individual difference factors. Teachers' personal accomplishment had the strongest effect on student perceptions. All three types of OCB impacted at least one form of student satisfaction.

\section{Outcomes of within and cross-domain support for work and family}

HASE, N., \& THOMPSON, B. (Griffith University)

b.thompson@griffith.edu.au

$T$ The proposal that supervisors and partners provide different types of support for work and family roles, within-domain support and otherdomain support, was tested by examining the relationship between supervisor and partner support for work and home and work-family conflict (WFC), work satisfaction and relationship satisfaction. 124 participants completed a questionnaire. Findings show that social support was negatively related to WFC and positively related to both work and relationship satisfaction. Women reported more support for work from supervisors and from partners than men. Other-domain support was a better predictor of outcomes than within-domain support, with partner support for work being the best predictor of WFC, FIW, and relationship satisfaction. The findings provide initial support for conceptualising unique types of support, and argue that a distinction between within-domain support and other-domain support is necessary for a more thorough understanding of the social support construct and its determinants.

\section{Alcohol and drugs project}

HO, B. (University of Queensland). bonnieho@psy.uq.edu.au

A communication audit was conducted by Project A Team A (Kieren Moffat, Anna Stallard \& Nathan Warner) examining communication between local alcohol and drug (A\&D) units and general practitioners (GPs). Methods of data collection involved focus groups and semi-structured interviews for GPs. Communication audit surveys 
were distributed to both groups. Results identified the reluctance of GPs to accept patients with substance abuse issues, due to concerns that participation in $A \& D$ training may attract excessive $A \& D$ referrals. Other obstacles included limited access to $A \& D$ information, lack of knowledge about services available, and a perception that $A \& D$ patients were difficult to deal with in a general practice environment. However, findings also indicate that $A \& D$ staff and GPs share a common interest in improving communication to overcome these obstacles. A number of recommendations were made, and the first stage of implementation is proceeding with Project Team B (Bonnie Ho \& Linda Chia) in consultation with Divisions of General Practice and A\&D staff.

\section{Predicting work performance and deviance from a biosocial model of personality}

JACKSON, C. (University of Queensland).

$\mathrm{T}$ ypical modern models of personality such as those of Eysenck's and the Big Five are hierarchical and are therefore more descriptive than explanatory. I develop an alternative 'biosocial model' of personality that proposes personality has its roots in biologically based 'instincts' which are then cognitively modified. I begin with Cloninger's ideas of personality and, by a series of rules, develop a series of paths that may explain as well as describe personality. This new model of personality predicts self-reported work performance, work deviance and other work-place variables.

\section{Testing a lateralised disinhibition hypothesis of neuroticism: The moderating effect of aural preference on neuroticism in the prediction of work performance}

JACKSON, CJ. (University of Queensland).

Chrisj@psy.uq.edu.au

$\mathrm{T}$ he hypothesis that neuroticism interacts with aural preference (preferred-ear for listening) in the prediction of disinhibited behaviour is tested. The importance of aural preference rests on the assumption that it is a readily available proxy measure of contrahemispheric preference such that a left aural preference is indicative of right hemispheric preference and vice versa. Whilst neuroticism is usually associated with avoidance, the left hemisphere (right aural preference) acts to initiate approach behaviour and the result of approach tendencies together with neuroticism is disinhibited behaviour. Study 1 shows that disinhibition can be positively predicted from neurotics with a right aural preference. Study 2 shows meaningful and replicated significant aural preference $\mathrm{x}$ neuroticism interactions in the prediction of aurally focused work performance, such that right aural preference people show a positive relationship between neuroticism and work performance. Results confirm the importance of aural preference as a moderator of neuroticism in the prediction of real-world behaviours and provide a new model of disinhibition.

\section{Merging university departments: A case study on the interaction of leadership, restructuring and human relations}

JOERIN, SB. (University of Queensland and the University of Zurich).

joerin@psy.uq.edu.au

$M$ ergers have become 'popular' in Australian Higher Education institutions, with the intention of resource efficiency and creating larger organisational units. Curri (2002) observed that often such restructuring was realised only on a surface level, and a reversion to old structures was not uncommon. She concluded that a successful merger is based on a concerted effort in three key areas: leadership, restructuring and managing relations, and integrated these key areas in a basic model without further elaboration. My aim was to determine the theoretical appropriateness and practical applicability of this model. The key areas were clarified through a literature search that combined organisational psychology concepts, change management literature and university specific studies (e.g., Curri, 2002, Hay \& Fourie, 2002; Harman, 2002) on leadership and restructuring. The model was then applied in a case study, which strongly supported its practical usefulness and provided further clarification. The three key areas proved to be an effective means of structuring interviews and a staff survey, and communicating results. Clarifications included elaborate definitions of the key areas, a clearer distinction of organisational levels, and a differentiation of external forces as well as outcome variables.

\section{Factors determining the priority given to failure recovery tasks}

\section{KANSE, L. (University of Queensland).}

kkanse@humanfactors.uq.edu.au

$T$ raditionally, the focus of safety management has mainly been on prevention of errors and other failures. More recently, research has become available focusing on what can be done after failures have occurred, but before this leads to negative consequences. That research highlighted people's abilities to detect and further recover from failures. 
When confronted with the need for recovery, people have to reprioritise tasks. This study examined factors influencing how people do this. Failure scenarios were developed in which eight such factors were incorporated, in different combinations for each scenario. Two hundred and twenty-two operators from two chemical plants each indicated for sample of 16 of these scenarios which priority they would give to the corresponding recovery tasks. Experience and occupational self efficacy measures were also obtained for each operator. Multilevel techniques were used for data analysis. Urgency and importance were found to be crucial scenario-level factors in prioritisation. Therefore organisations should ensure their people are able to make correct assessments for these factors. Even though there were no main effects of self efficacy and experience, an interaction effect was found indicating that more efficacious people tend to delay recovery tasks further in favour of other urgent and important tasks than less efficacious colleagues.

\section{Learning to perform a novel task:} A multilevel analysis of negative affect, goal orientation, practice and performance

KOY, A., \& YEO, G. (University of Queensiand). koy@psy.uq.edu.au

$\mathrm{T}$ This study examines the relationship between negative affect and performance during skill acquisition. Participants were given multiple practice trials on an air traffic control simulation task and were required to judge whether pairs of converging aircraft would conflict or pass safely. Prove- and avoid-orientation were measured prior to task performance. Negative affect and performance were measured at repeated intervals throughout practice. Hierarchical Linear Modelling (HLM) was performed to examine within-person relationships as well as interactions across two levels of analysis. As expected, there was a negative relationship between negative affect and performance at the within-person level, suggesting that increases in negative affect were associated with decreases in performance. A significant interaction between negative affect and practice was also found. Follow-up analyses revealed that the relationship between negative affect and performance strengthened across practice. Prove- and avoid- orientation did not influence the rate of performance improvement or moderate the relationship between negative affect and performance. The results of this study are interpreted using a resource allocation framework and the implications for training design discussed.

\section{Familial orientation and psychological contract breach in the Philippines: One big happy family?}

LLOYD RESTUBOG, S. (University of Queensland).

$T$ he nature of employment relationships in collectivist cultures has been described as familial and in some ways similar to a parental relationship. The present research consists of 2 studies aimed at examining the role of familial orientation in the context of psychological contract breach. In Study 1, we interviewed 30 employees to explore the nature of employment relationships and identify their expectations toward their employer. Results confirmed the nature of familial orientation and its role in employee-employer relationships. In Study 2, we surveyed 267 full-time employees to test the direct and moderating role of familial orientation on the effects of breach on civic virtue behaviour. We found evidence of main effects of familial orientation and breach of relational obligations on civic virtue behaviour. Familial orientation also moderated the relationship between breach and civic virtue behaviour, with the relationship between breach and civic virtue behaviour weaker when familial orientation was high. These findings suggest that familial orientation can serve as a social support resource capable of mitigating the negative effects of psychological contract breach on civic virtue behaviour.

Organisational culture, stress, and coping: Qualitative and quantitative exploration

NEWTON, C., \& JIMMIESON, N.

(University of Queensland).

c.newton@psy.uq.edu.au

$\mathrm{T}$ he Competing Values Framework (Quinn, 1988) identifies four types of organisational culture (i.e., human relations, open systems, rational goal and internal process). The purpose of this study was twofold - to identify the primary types of stressors experienced by employees in each organisational culture, and to determine the types of coping strategies typically used by employees to deal with these stressors in their workplace. Purposeful sampling was utilised with organisations selected based on a priori expectations about their dominant culture type. In order to reduce the impact of individual job characteristics on the reported results, all organisations were retailbased, with all interviewees involved in client service activities. In all, 60 employees from 5 client-focused organisations were interviewed to determine common and distinct stressors and coping strategies. Analysis of the results revealed a pattern of differences between each of the organi- 
sational culture typologies in terms of the types of stressors experienced by employees in their workplace. Similarly, the types of emotionfocussed coping strategies employees used to deal with workplace stressors differed according to organisational culture.

\section{Hierarchy of abstraction modelling as a research method and means of presenting research findings}

PARRY, K. (Griffith University).

$\mathrm{T}$ he hierarchy of abstraction model (H.A.M.) is an important outcome of grounded theory research. It is also an important learning and sensemaking tool for researchers during the process of research. Finally, it is also an important learning tool for participants in executive development programs. This modelling is also represented in a modified form as the outcome of structural equation modelling. This paper will take you through some experience the author has had with grounded theory research and psychometric modelling, and will pose the benefits to be gained from using this modelling as a research tool. The author will also attempt to show how the insights to be gained from this work can be of benefit to those people schooled in purely qualitative research as well as those schooled in psychometric research.

\section{An examination of the relationship between conscientiousness and citizenship performance and task performance, within an academic setting}

\section{POROPAT, A. (Griffith University)}

arthur.poropat@griffith.edu.au

W hen initially formulated, citizenship oerformance, and its earlier labels of organisational citizenship and contextual performance, was proposed to reflect noncognitive aspects of the workplace context, and of individual workers, especially the personality dimension of conscientiousness. Despite this, research examining the relationship between conscientiousness and citizenship, including several meta-analytic studies, has typically found only modest correlations between conscientiousness and citizenship performance. However, there is evidence to suggest that the manner in which personality is measured moderates the strength of correlations between personality and performance measures. In particular, personality ratings provided by persons, other than the ratee, have been more strongly related to performance ratings than self-ratings of personality. Consequently, this study examined the relationship between self-rated and other-rated conscientiousness, and both citizenship and task performance. Participants were first-year undergraduate students, with relatively brief acquaintance with each other. Despite this, when compared with selfratings of Conscientiousness, other-ratings of conscientiousness, provided by fellow students, were observed to be much more strongly related to both citizenship and task performance, even when the respective ratings were provided by independent raters. Implications of these findings for understanding both conscientiousness and citizenship performance are discussed.

\section{School leadership, culture, and teacher stress: Implications for problem students}

QUINN, AJ. (University of Queensland).

a.quinn@psy.uq.edu.au

$\mathrm{C}$ ontextual factors linked to student behaviour problems in schools include leadership, organisational culture (within individual schools), and levels of teacher stress. The aim of this study concerns the direction of effects from school level variables and offers an alternative perspective on the environment-behaviour equation. That is, while student misbehaviour is viewed as a 'producer' of teacher stress, it could also be a 'product'. Participants were teaching staff $(N=153)$, school administrators $(N=17)$ and students referred for behavioural problems $(N=1432)$ at seven Brisbane metropolitan schools. Teachers and school administrators completed the Multifactor Leadership Questionnaire and the Organisational Culture Inventory, while teachers also completed the Maslach Burnout Inventory. Student data was collated from school records, and grouped according to referral frequency per student. High referral rates were associated with transactional leadership and an oppositional aspect to organisational culture. Low and medium referral rates were associated with transformational leadership and dependent, approval-oriented, and affiliative aspects of culture. Teacher stress did not account for substantial variance in referral rates for misbehaviour. Results supported the hypothesis that school environment variables may be important in approaches to behaviour management. 
The cultural influence and consumer attitude toward the usage of sexual appeal in advertising: Comparative study among Asians, Asian-Americans, and white Americans

SAWANG, S. (University of Queensland).

sawang@psy.uq.edu.au

A dvertising across cultural boundaries is a difficult and challenging task. The advertising is heavily influenced by cultures. The complexities of the term 'culture' encompasses a wide range which includes knowledge, belief, art, law, morality, customs, along with any other capabilities and habits acquired by humans as members of society. The operation of culture is based on setting loose boundaries for individual behaviour, as well as by influencing the functioning of the consumer and mass media; thus, a successful advertising campaign should take into account the existing cultural differences inherent to that society. The effects of sexoriented advertisements on the buying public, based on the particular culture(s) involved in Asians, Asian-Americans, and white Americans were investigated in number of 60 participants. The type of measurement used was an interval level measurement, based on $\mathrm{Q}$ Methodology. The findings are that White-Americans are more likely to prefer the use of sex appeal in advertisement than they are when nothing sexually explicit is used. However, Asian participants are more likely to prefer it when there is less usage of sexual appeal in advertisements. For Asian-Americans, they tend to agree with American participants regarding the usage of sex appeal in advertisement.

\section{On being different: Understanding diversity influences on employee wellbeing and team functioning}

SHEEHAN, A., \& MARTIN, R.

(University of Queensland).

annash@psy.uq.edu.au

$\mathrm{D}$ iversity refers to the extent to which people perceive they differ. The objectives of this research were twofold: to extend current research by (a) examining the impact of perceived diversity on team functioning and (b) to examine the moderating role of diversity tolerance on the perceived diversity-team conflict relationship. Study 1 sought to determine what dimensions of diversity were perceived as important in impacting on team functioning in firefighters. A total of 28 semi-structured interviews were conducted. Content analyses revealed three important diversity dimensions: surface; job related; values, attitudes and beliefs
(VAB). In Study 2, a survey was sent to all firefighters in one Australian state. A total of 539 surveys were returned (response rate $36 \%$ ). Confirmatory factor analysis supported the three factor structure of diversity. A series of multiple regressions found that VAB diversity, which is rarely examined in the literature, had a significant effect above surface and job-related diversity in relation to team conflict, wellbeing, intrinsic satisfaction and team commitment. A moderated multiple regression found that participants with a lower diversity tolerance were more negatively affected when VAB diversity was perceived.

\section{Intergroup relations during organisational mergers}

\section{TERRY, D. (University of Queensland).}

$T$ his paper will overview a program of research that has been designed to examine, from a social identity perspective, the predictors of employee responses to an organisational merger. Both field and experimental research will be outlined in support of a social identity approach to the understanding of the impact of an organisational merger. The field research comprises both cross-sectional and longitudinal studies of private and public sector organisations involved in organisational mergers. Each of these studies has shown that, in support of a social identity approach, ingroup bias is prevalent in the context of an organisational merger, and that the nature of this bias accords with predictions. Moreover, there is support for the moderating role of socio-structural beliefs in the prediction of employee responses to a merger. The experimental research has focused on group-level factors designed to facilitate success of a merger - these include the group membership of the assigned leader of the new group and the nature of the integration pattern.

\section{Innovation: Is it all it's cracked up to be?}

UNSWORTH, K. (Queensland University of Technology).

$I^{\prime}$ nnovation is often touted as the panacea for the dynamic, changing marketplace that we currently find ourselves in. Governments at all levels are calling for greater innovation (e.g., 'Smart State'), and academics are heeding that call. Numerous studies have identified factors that increase innovation, but few have looked at the consequences of innovation. This talk presents results from two studies that indicate that innovation may, in fact, have potentially negative outcomes for individuals and organisations. The first study, focusing on individuals, employed a qualitative methodology: 
data was collected from four sites across two companies, including interviews with 65 engineers, four-week long diary studies of 25 engineers, and observational studies. Findings suggest that both generating and implementing ideas is difficult, timeconsuming, and often punished by the organisation. The second study focused on the effect of organisational innovation. Data from AWIRS showed that organisations that implemented innovations increased their profit five years later only when the relationship between management and workers was positive. When this relationship was negative, there was a decrease in economic performance after implementing innovation. It is likely that innovation is not a cure-all, and that certain conditions must be in place before the benefits can be realised. 